\section{Paradoxical results in and a possible extension of Necessary Condition Analysis (NCA)}

Kimmo Sorjonen ${ }^{1}, \&$ Bo Melin ${ }^{1}$

${ }^{1}$ Department of Clinical Neuroscience, Karolinska Institutet, Stockholm, Sweden

Abstract

Necessary Condition Analysis (NCA) is a quite new statistical method that has been used, for example, to analyze the association between intelligence and creativity. However, a significant result in NCA only indicates that the association between two variables, $\mathrm{X}$ and $\mathrm{Y}$, is characterized by some unspecified type of non-randomness, and not necessarily that $\mathrm{X}$ is necessary for $\mathrm{Y}$. The present simulation showed that the significance of necessity effects, as calculated through permutation in NCA, tend to increase with increased degree of sufficiency, even when the increase in sufficiency is achieved through decreasing the sample size. This is paradoxical for a method whose stated objective is to help researchers identify necessary but not sufficient conditions for some outcome of interest. One possible way to increase the specificity and usefulness of results obtained through NCA is to calculate not only the degree of necessity but also the degree of sufficiency and the difference between these two. The significance of the difference between degree of necessity and sufficiency can be estimated through bootstrapping. The simulation indicated desirable statistical characteristics for this extended version of NCA and it was applied on empirical data on intelligence, creative ability, and creative achievement.

Keywords: creativity; intelligence; necessary condition analysis; necessity; specificity; sufficiency

\section{Introduction}

In Necessary Condition Analysis (NCA), a necessity effect is estimated by calculating the amount of empty space in the upper left corner in a $\mathrm{X}$-Y-plot as a percentage of the size of the square given by the lowest and the highest $\mathrm{X}$ and $\mathrm{Y}$ values. An empty space is taken to indicate that a certain degree of $\mathrm{X}$ is necessary for a high value on $\mathrm{Y}$. Values below 0.1 have been characterized as small, values between 0.1 and 0.3 as medium, values between 0.3 and 0.5 as large, and values above 0.5 as very large necessity effects (Dul, 2016). Researchers employing NCA have concluded, for example, that a certain (not too low) degree of intelligence is necessary but not sufficient for a high degree of creativity (Karwowski et al., 2016, 2017; Shi et al., 2017) and that academic achievement in math is a necessary condition for creativity in math (Karwowski et al., 2020).

However, Sorjonen, Wikström Alex, and Melin (2017) demonstrated that strong necessity effects can be observed with a negatively skewed $X$ and a positively skewed $\mathrm{Y}$ even if the two variables have no association with each other (see Ilagan \& Patungan, 2018, for additional counterexamples). Dul, van der Laan, and Kuik (2020) argued that such necessity effects that are due to pure randomness can be detected through calculation of p-values with so called permutation tests and this feature has been included in later versions of the NCA package in R (Dul, 2021).

But, as Dul, van der Laan, Kuik, and Karwowski (2019) pointed out, this significance test in NCA is only capable of assessing if the association between $\mathrm{X}$ and $\mathrm{Y}$ is characterized by randomness or not. This means that a significant result only indicates that the association between $\mathrm{X}$ and $\mathrm{Y}$ is characterized by some unspecified type of non-randomness, and not necessarily that $\mathrm{X}$ is necessary for $\mathrm{Y}$. In accordance with this lack of specificity, Sorjonen and Melin (2019) showed that for a certain degree of true necessity effect in the population, the significance of results as calculated through permutation in NCA actually tend to increase (i.e. the p-value tends to decrease) as the true degree of sufficiency in the population increases. As Sorjonen and Melin (2019, p. 4) noted, this:

is paradoxical for a method whose objective is to identify necessary but not sufficient conditions for an outcome $Y$. As insufficiency is a necessary condition for "necessary but not sufficient" the significance of results from NCA should, on the contrary, decrease with increased degree of sufficiency.

It is probably safe to say that the main rationale for significance tests is to help researchers avoid erroneous conclusions, for example claiming that there is a correlation between $\mathrm{X}$ and $\mathrm{Y}$ when there actually is not. The certainty of a true correlation between $\mathrm{X}$ and $\mathrm{Y}$ increases with: (1) The strength of the correlation in the sample, and (2) the size of the sample. Similarly, the certainty of true differences in risks and means and adjusted regression effects increase: (1) The more the calculated difference or effect in the sample deviates from the value that indicates no difference/effect, and (2) with the sample size.

As mentioned above, the stated objective of NCA is to help researchers identify conditions that are necessary but not sufficient for a certain outcome Y. Given this objective, and in accordance with the reasoning above, it would be desirable if the significance of results in NCA would increase, i.e. the $\mathrm{p}$-value decrease, the more confident one can be that $\mathrm{X}$ is necessary but not sufficient for high levels 

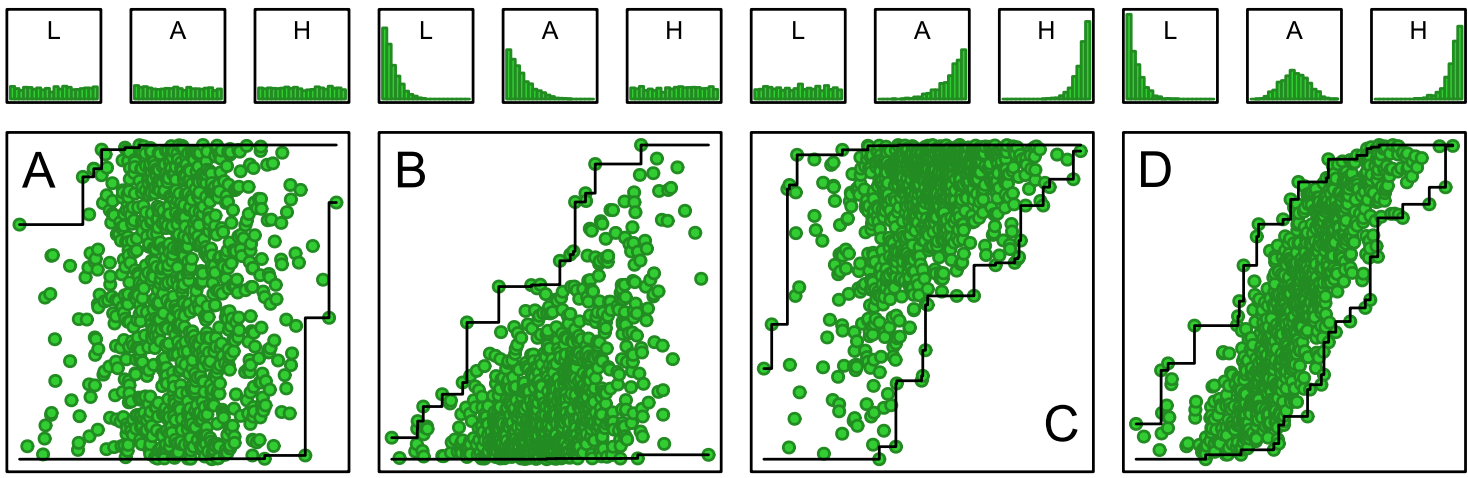

Figure 1. Top row: The distribution that Y-values were drawn from for those with the lowest (L), average (A), and highest $(\mathrm{H}) \mathrm{X}$-value in the plots beneath. Bottom row: When subjects were assigned Y-values from a uniform distribution irrespective of their X-value data tended to exhibit neither necessity nor sufficiency (Panel A). When the distribution that $\mathrm{Y}$-values were assigned from changed from positively skewed to uniform a high degree of necessity could be observed (Panel B), when it changed from uniform to negatively skewed a high degree of sufficiency (Panel C) could be observed, and with a change from a positively to a negatively skewed distribution both necessity and sufficiency (Panel D) could be observed. X-values have been drawn from a random normal distribution $(N=1000)$.

of Y. This means that the significance of results in NCA should increase: (1) When the size of the necessity effect in the sample increases; (2) when the size of the sufficiency effect in the sample decreases; and (3) when the sample size increases.

Sorjonen and Melin (2019) showed that the probability for a significant result in NCA increases with an increase in the necessity effect, i.e. that criterion (1) above seems to be fulfilled. The objective of the present study was to evaluate if analyses in NCA fulfill the two other desirable statistical characteristics mentioned above. Also, an extended version of NCA that increases the specificity and usefulness of results obtained through NCA by calculating not only the degree of necessity but also the degree of sufficiency and the difference between these two will be presented.

\section{Methods}

In order to evaluate if the significance test in NCA possess the desirable characteristics (2) and (3) mentioned above, a simulation with $\mathrm{R}$ 4.1.0 statistical software (R Core Team, 2021) employing the NCA package (Dul, 2021) was conducted. Script and data are available at https://osf.io/ek9ny/. Two random uniform variables $(N=1000)$ were created and then increasingly large chunks of observations were deleted from the lower right corner of the bivariate distribution. As this manipulation both increases the degree of sufficiency (operationalized as the size of the empty space in the lower right
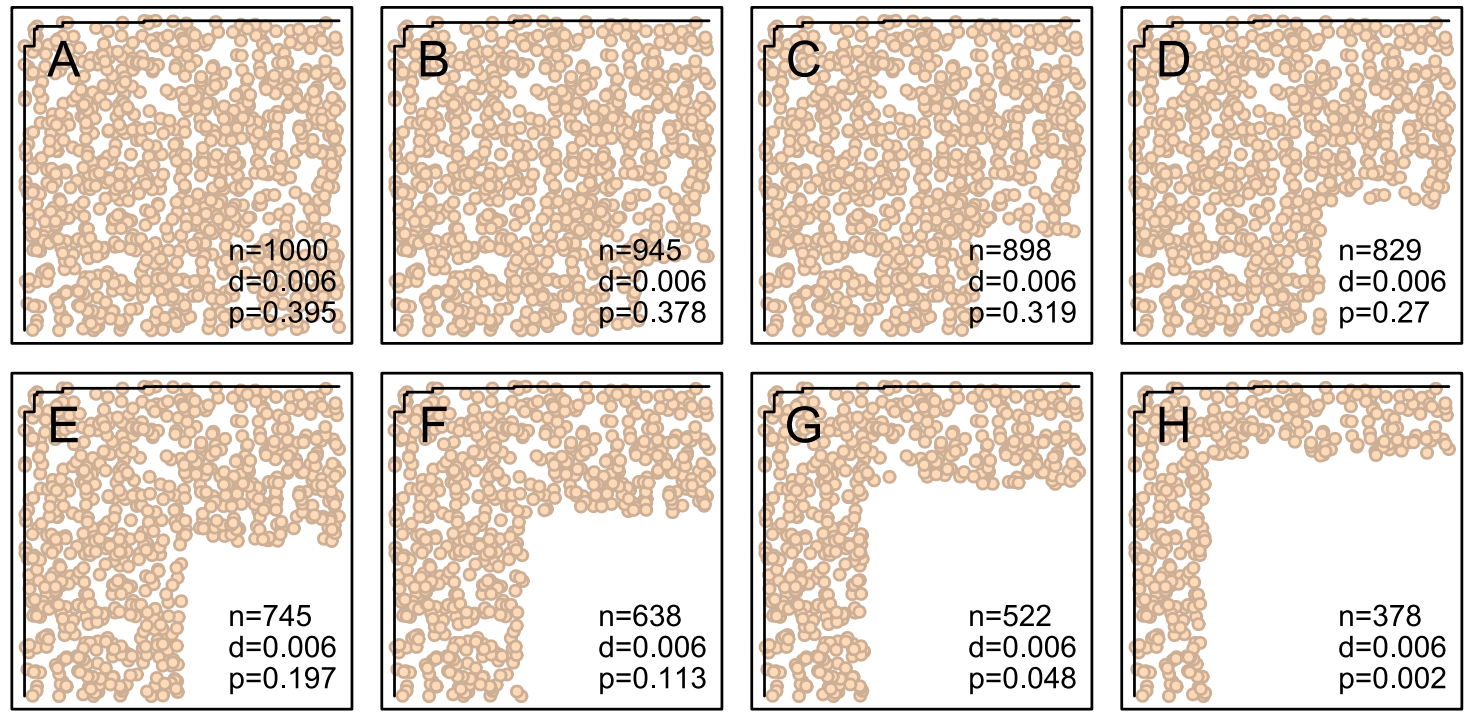

Figure 2. The size (d) and significance (p) of the necessity effect between two variables drawn from a random uniform distribution, as calculated through permutation in NCA, as functions of deleting increasingly large chunks of observations in the lower right corner, thereby increasing the degree of sufficiency and decreasing the sample size. 
corner) and decreases the sample size, it decreases the certainty of $X$ being necessary but not sufficient for a high level of Y. Therefore, this manipulation should decrease the significance of the necessity effect, i.e. increase the $\mathrm{p}$-value.

In the next step, necessity and sufficiency were simulated by assigning virtual participants Y-values from different distributions depending on their $\mathrm{X}$ value, which they were assigned from a random normal distribution. If the Y-distribution is gradually changed from positively skewed to uniform with increasing $\mathrm{X}$-value, data tend to exhibit necessity (Figure 1, Panel B). If the gradual change is from uniform to negatively skewed, data suggest sufficiency (Panel C) and if applying both of these gradual changes simultaneously, data exhibits both necessity and sufficiency (Panel D). The degree of necessity and sufficiency can be manipulated by making the positively and negatively skewed distributions more or less extreme. In Panel B in Figure 1 the gradual change is from a positively skewed beta-distribution with alpha $=1$ and beta $=9$ to a uniform beta-distribution with alpha $=1$ and beta $=1$. With a more extreme starting point, e.g. alpha $=1$ and beta $=100$, the size of the empty space in the upper left corner would, with very high probability, have been larger and the calculated necessity effect stronger. Similarly, the degree of sufficiency in Panel C could have been increased by assigning those with the highest $\mathrm{X}$-values $\mathrm{Y}$-values from a beta-distribution with alpha $=100$ and beta $=$ 1 instead of, as now, alpha $=9$ and beta $=1$.

15840 samples with different combinations of degree of necessity (max beta either 1,3 , 9, or 27 as described above), degree of sufficiency (max alpha either 1, 3, 9, or 27), and sample size (between 3 and

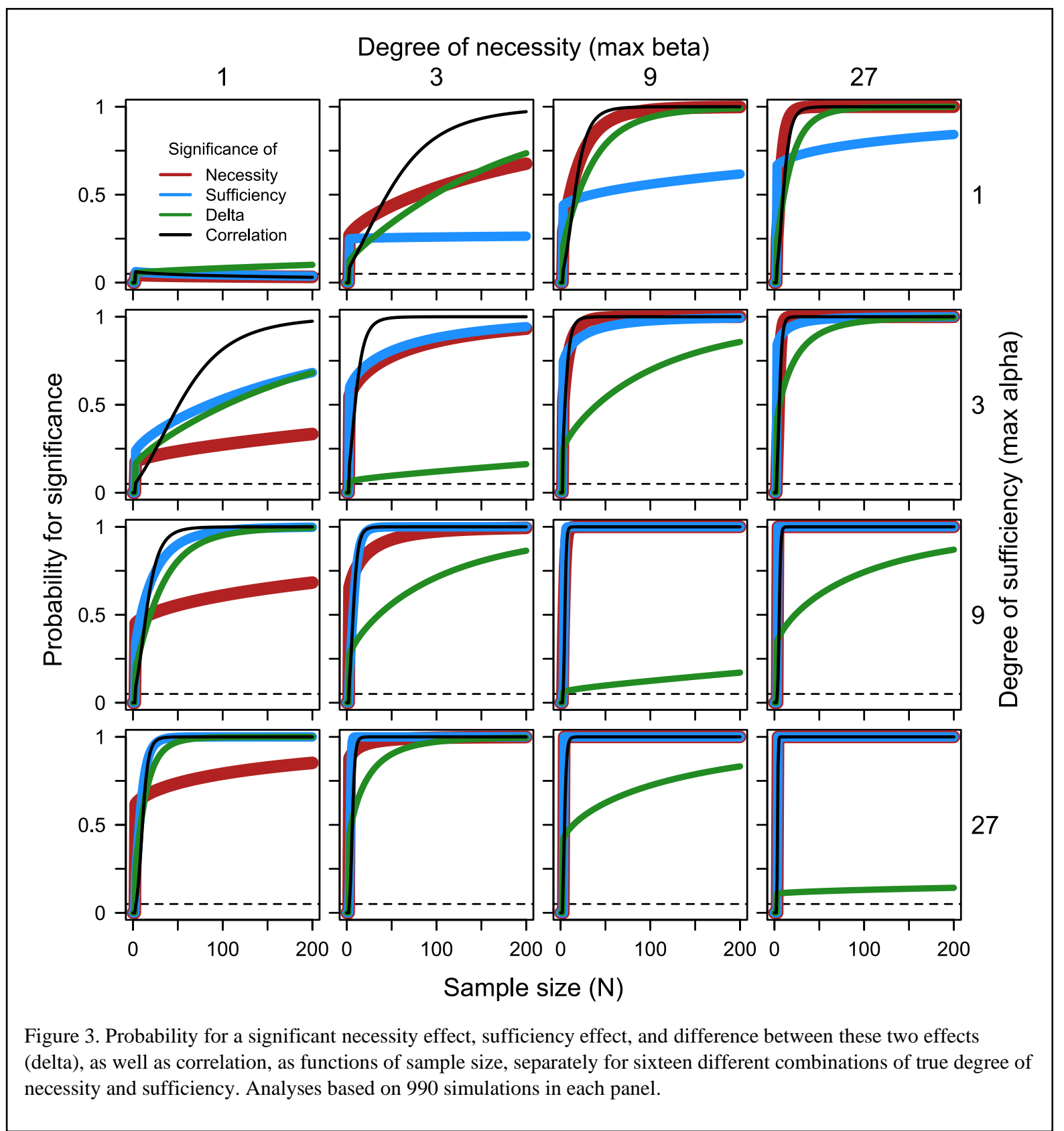


200) were created. In each sample, the size and significance of the necessity and the sufficiency effects were calculated, as well as the difference between these effects in 1000 bootstrapped subsamples. Although NCA does not calculate sufficiency effects, this can be achieved by reversing $\mathrm{X}$ and $\mathrm{Y}$, as the necessity effect of reversed $\mathrm{X}$ on reversed $Y$ equals the sufficiency effect of original $\mathrm{X}$ on original $\mathrm{Y}$. The $Z$-score, and then the $p$-value, of the difference was calculated by dividing the mean with the standard deviation of the 1000 bootstrapped differences. The association between the square root of the sample size and the probability for a significant $(p<.05)$ necessity effect, sufficiency effect, and difference between these two was calculated through logistic regression separately for the 16 different combinations of degree of necessity and degree of sufficiency.

\section{Results}

As it should be, only a very weak and nonsignificant necessity effect between two random uniform variables was found (Figure 2, Panel A). Although it might be hard to spot, there is a tiny empty space in the upper left corner in the plots in Figure 2. This empty space is due to the fact that the probability to get an observation that combines the lowest value on $\mathrm{X}$ and the highest value on $\mathrm{Y}$ usually is very low, even if there is no necessity involved in the association between $\mathrm{X}$ and $\mathrm{Y}$.

As mentioned above, deleting increasingly large chunks of observations from the lower right corner of the bivariate distribution decreases the certainty of $\mathrm{X}$ being necessary but not sufficient for a high level of $\mathrm{Y}$ and should, therefore, decrease the significance of the necessity effect, i.e. increase the $p$-value. However, as seen in Panels B to $\mathrm{H}$ in Figure 2 , the effect of this manipulation was exactly the opposite - it increased the significance of the necessity effect.

Figure 3 shows, again and paradoxically, that the probability for a significant necessity effect, as calculated through permutation in NCA, increased with an increase in the sufficiency effect (the steepness of the red curve increases for each panel if moving downward in a specific column). This was the case even if the true degree of necessity equaled zero (leftmost column). Similarly, the probability for a significant sufficiency effect increased with degree of necessity (the steepness of the blue curve increases for each panel if moving to the right in a specific row). As a comparison, the probability for a significant correlation is indicated in the panels in Figure 3 . With a few exceptions, with very small sample sizes, a correlation was at least as good, and in some cases better, at identifying a true necessity/sufficiency effect as the significance test in NCA (the black curve is above the red and blue curves).
The significance of the difference between the necessity and the sufficiency effect, calculated through bootstrapping, seemed to behave quite well. The probability for a significant result increased, as it should, with increased sample size and the size of the true difference, as indicated by an increase in the steepness of the green curve for each panel when moving right/left or up/down from the main diagonal in Figure 3 (the true difference is largest in the upper-right and the lower-left panel). However, there seemed to be some inflation of the risk for type 1-error above the nominal 5\% when no true difference was present, especially if both the true necessity and the true sufficiency effects were strong (see the main diagonal in Figure 3). Hence, it might be preferable to use a more conservative significance level, e.g. .01, than the traditional .05 if using this method.

\section{Warning}

As shown by Sorjonen et al. (2017), a negatively skewed $\mathrm{X}$ and a positively skewed $\mathrm{Y}$ variable can often be enough to observe a strong necessity effect and with such data there was also a high probability to observe a significant difference between the necessity and the sufficiency effect, as calculated through bootstrapping (Figure 4). The same is true with a positively skewed $\mathrm{X}$ and a negatively skewed $\mathrm{Y}$, which tends to result in a strong sufficiency effect. Hence, the necessity (sufficiency) effect should be significant, as calculated through permutation in NCA, if concluding that it is stronger than the sufficiency (necessity) effect, as calculated through bootstrapping.

\section{Empirical application}

The presented extended version of NCA was applied on a selection of data used by Jauk, Benedek,

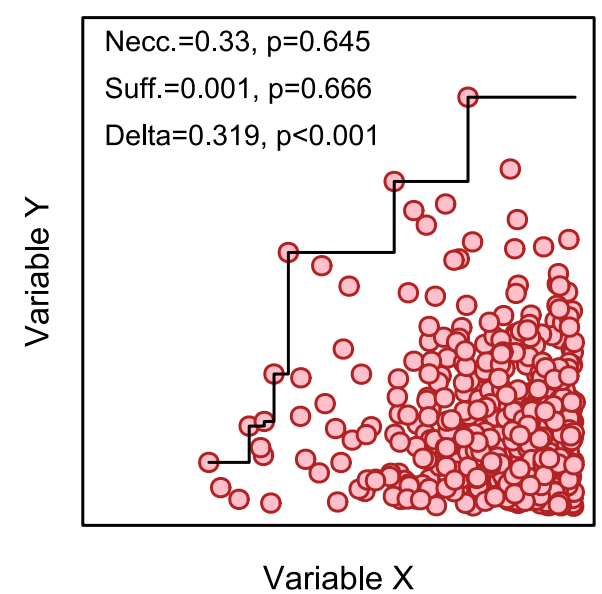

Figure 4. A significant difference (delta) between necessity and sufficiency that was due to a negatively skewed $\mathrm{X}$ and a positively skewed $\mathrm{Y}$ variable. 


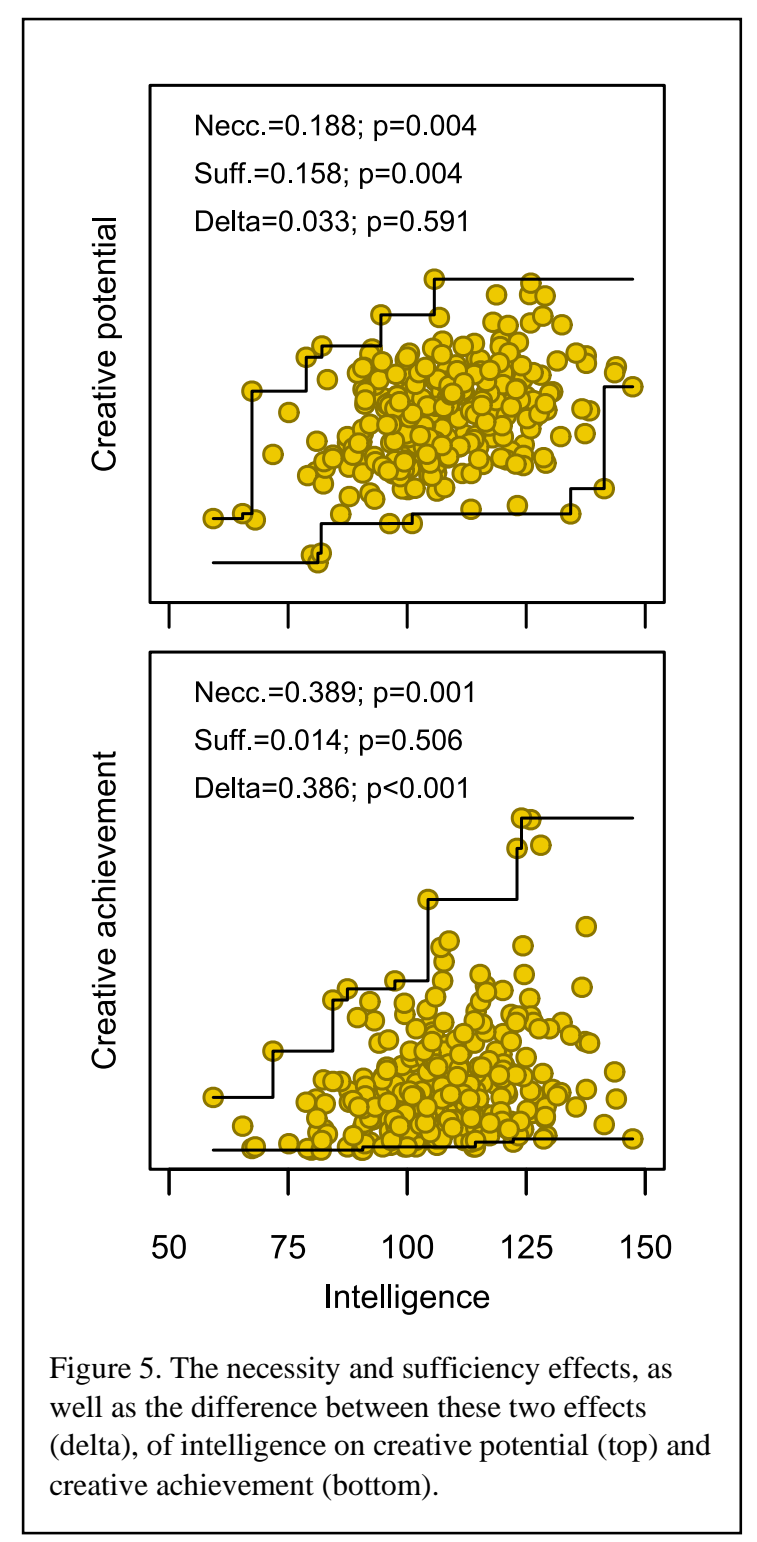

Dunst, and Neubauer (2013), who collected data on subjects' $(N=297)$ intelligence, creative potential (average originality), and creative achievement. Based on the results in Figure 5 it might be concluded that the association between intelligence and creative potential is characterized by equal degrees of necessity and sufficiency while the association between intelligence and creative achievement is characterized more by necessity than by sufficiency. Using a different method, Ilagan and Patungan (2018) reached a similar conclusion.

\section{Discussion}

The present simulations indicate that the significance of necessity effects, as calculated through permutation in NCA, tend to increase with increased degree of sufficiency, even when the increase in sufficiency is achieved through decreasing the sample size. Given enough sufficiency even the tiniest necessity effect, completely due to randomness, can become significant. This is paradoxical for a method whose stated objective is to help researchers identify necessary but not sufficient conditions for some outcome of interest.

It can be assumed, or at least hoped, that researchers would be reluctant to conclude that $\mathrm{X}$ is necessary but not sufficient for $\mathrm{Y}$ if they, for example, receive data resembling those in Panel $\mathrm{H}$ in Figure 2, even if the significance test in NCA signals a highly significant finding. As Dul et al. (2019) conclude, this significance test only evaluates if the association between $\mathrm{X}$ and $\mathrm{Y}$ is characterized by some unspecified type of nonrandomness and not if $\mathrm{X}$ can be assumed to be necessary for Y. Most researchers would probably find it quite unsatisfactory to be limited to such a blunt conclusion.

Dul et al. (2019) argue that the tendency for NCA to give significant results when $\mathrm{X}$ is sufficient but not necessary for Y, i.e. the exact opposite of what NCA was designed to detect, is not a problem. Actually, they think this is a desirable characteristic as it, in their words, is an indication of the power of the test. Given the stated objective to identify conditions that are necessary but not sufficient for the outcome, we find this statement a bit odd. Statistical tests, as well as other appliances, should be devised to accomplish a specified task, instead of specifying the task based on what the device happens to accomplish. The argument by Dul et al. is a bit like constructing an algorithm for identifying promising job applicants, defined as having a lot of experience but not too high salary demands. When it turns out that the algorithm picks out applicants with a lot of experience OR with a very high salary demand (given a high enough salary demand, they do not need any experience in order to be identified as promising) we argue that this is a good thing because the algorithm is a randomness test and cannot test for a specific alternative hypothesis. Clearly, if the algorithm does not do what we intended, it should be described as flawed. Besides, if Dul et al. just wants a test that is good at identifying non-randomness, they could make do with a simple correlation, as it seems better at the job than NCA (in Figure 3, the black lines tend, if there is any difference, to be above the red lines).

One way to increase the specificity and usefulness of results obtained through NCA is to calculate not only the degree of necessity but also the degree of sufficiency and the difference between these two. The significance of this difference can be estimated through bootstrapping and the simulations demonstrated that the probability for a significant difference increases with increased sample size and the size of the true difference, which is desirable for a test of statistical significance. However, in the present simulation the method exhibited some inflation in the risk for type 1-errors, which might call for the use of a more conservative significance level than the traditional .05. Moreover, the method 


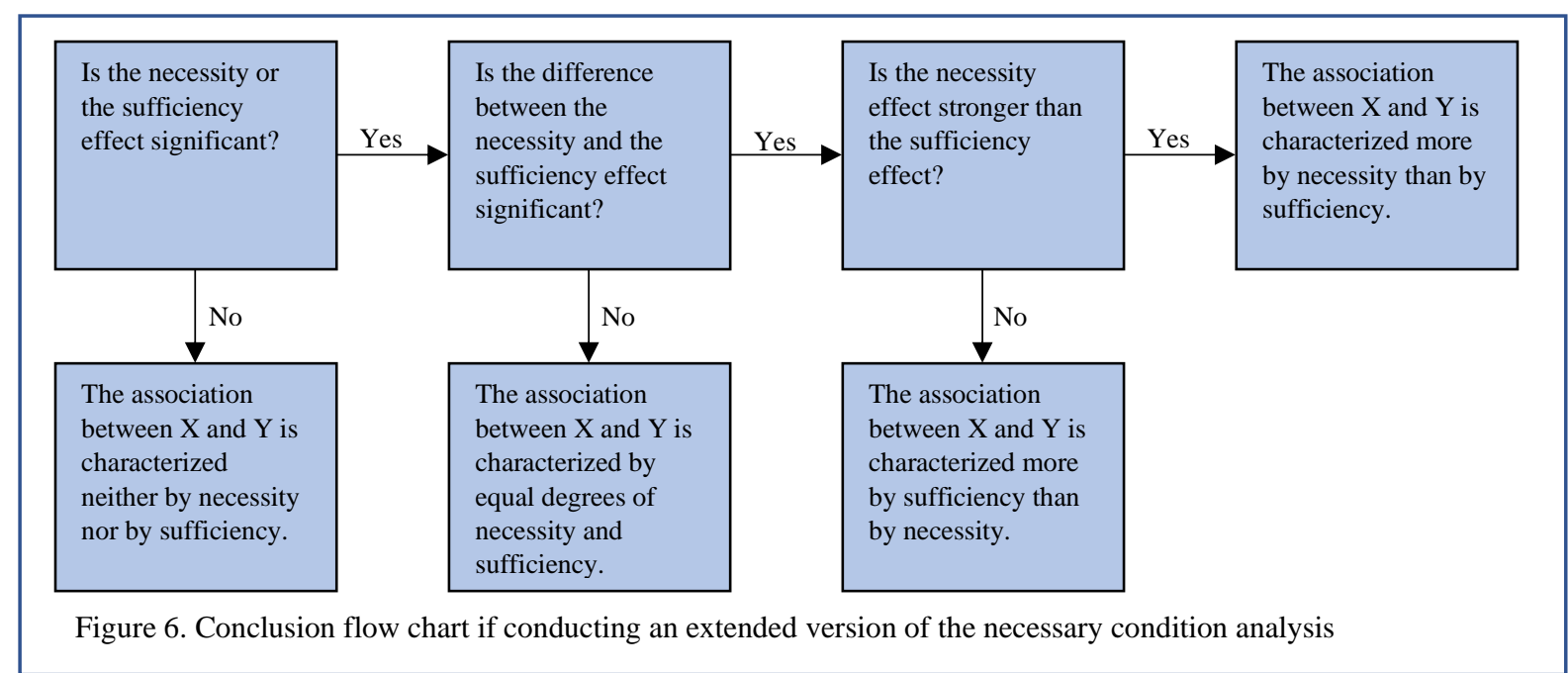

can indicate a significant difference that is solely due to skewness. Therefore, one should refrain from concluding that the necessity (sufficiency) effect is stronger than the sufficiency (necessity) effect unless it has been found to be significant through the permutation test in NCA.

If researchers choose to use the presented "extended version of NCA", for example because they wish to draw more specific conclusions than that the association between $\mathrm{X}$ and $\mathrm{Y}$ is characterized by some unspecified type of nonrandomness, which is what the original version of NCA offers, the flow chart in Figure 6 can be used as an aid.

\section{Conclusions}

The significance of necessity effects, as calculated through permutation in NCA, tend to increase with increased degree of sufficiency, even when the increase in sufficiency is achieved through decreasing the sample size. Given enough sufficiency even the tiniest necessity effect, completely due to randomness, can become significant. This is paradoxical for a method whose stated objective is to help researchers identify necessary but not sufficient conditions for some outcome of interest. To calculate the difference between necessity and sufficiency effects through bootstrapping seems like a promising method in order to increase the specificity and usefulness of results obtained through NCA. The probability for a significant difference increases with increased sample size and the size of the true difference, which is desirable for a test of statistical significance. However, one should refrain from concluding that the necessity (sufficiency) effect is stronger than the sufficiency (necessity) effect unless it has been found to be significant through the permutation test in NCA.

\section{Acknowledgements}

We would like to thank Jauk et al. (2013) for making their data available at https://osf.io/hzpgy/
Data availability

Data and script for the simulations are available at Open Science Framework, https://osf.io/ek9ny/. Data for the empirical analyses are available at https://osf.io/hzpgy/.

\section{References}

Dul, J. (2016). Necessary Condition Analysis (NCA): Logic and methodology of "necessary but not sufficient" causality. Organizational Research Methods, 19(1), 10-52.

https://doi.org/10.2139/ssrn.2588480

Dul, J. (2021). NCA: Necessary Condition Analysis. $R$ package version 3.1.1. Https://CRAN.Rproject.org/package $=$ NCA.

Dul, J., van der Laan, E., \& Kuik, R. (2020). A statistical significance test for Necessary Condition Analysis. Organizational Research Methods, 23(2), 385-395. https://doi.org/10.1177/1094428118795272

Dul, J., van der Laan, E., Kuik, R., \& Karwowski, M. (2019). Necessary Condition Analysis: Type I error, power, and over-interpretation of test results. A reply to a comment on NCA. Commentary: Predicting the significance of necessity. Frontiers in Psychology, 10, 1493. https://doi.org/10.3389/fpsyg.2019.01493

Ilagan, M. J., \& Patungan, W. (2018). The relationship between intelligence and creativity: On methodology for necessity and sufficiency. Archives of Scientific Psychology, 6, 193-204. https://doi.org/10.1037/arc0000050

Jauk, E., Benedek, M., Dunst, B., \& Neubauer, A. C. (2013). The relationship between intelligence and creativity: New support for the threshold hypothesis by means of empirical breakpoint detection. Intelligence, 41, 212-221. https://doi.org/10.1016/j.intell.2013.03.003

Karwowski, M., Dul, J., Gralewski, J., Jauk, E., Jankowska, D. M., Gajda, A., Chruszczewski, M. H., \& Benedek, M. (2016). Is creativity without intelligence possible? A Necessary Condition Analysis. Intelligence, 57, 105-117. https://doi.org/10.1016/j.intell.2016.04.006

Karwowski, M., Jankowska, D. M., Brzeski, A., Czerwonka, M., Gajda, A., Lebuda, I., \& Beghetto, R. A. (2020). Delving into creativity and learning. 
Creativity Research Journal, 32(1), 4-16.

https://doi.org/10.1080/10400419.2020.1712165

Karwowski, M., Kaufman, J. C., Lebuda, I., Szumski, G., \& Firkowska-Mankiewicz, A. (2017). Intelligence in childhood and creative achievements in middle-age: The necessary condition approach. Intelligence, 64 36-44. https://doi.org/10.1016/j.intell.2017.07.001

$\mathrm{R}$ Core Team. (2021). $R$ : A language and environment for statistical computing. $R$ Foundation for Statistical Computing, Vienna, Austria. URL https://www.Rproject.org/.
Shi, B., Wang, L., Yang, J., Zhang, M., \& Xu, L. (2017). Relationship between Divergent Thinking and Intelligence: An Empirical Study of the Threshold Hypothesis with Chinese Children. Frontiers in Psychology, 8 . https://doi.org/10.3389/fpsyg.2017.00254

Sorjonen, K., \& Melin, B. (2019). Predicting the significance of necessity. Frontiers in Psychology, 10, 283. https://doi.org/10.3389/fpsyg.2019.00283

Sorjonen, K., Wikström Alex, J., \& Melin, B. (2017). Necessity as a function of skewness. Frontiers in Psychology, 8, 2192.

https://doi.org/10.3389/fpsyg.2017.02192 